\title{
Pulmonary valve stenosis due to undifferentiated pleomorphic sarcoma
}

\section{Panagiotis Antiochos ${ }^{a}$, Pierre Monneya, Salah Dine Qanadlic, Leopold Schlueter ${ }^{e}$, Samuel Rotman ${ }^{b}$, Emilienne Descloux ${ }^{b}$, Lars Niclauss ${ }^{d}$, Michel Hurni $^{d}$, Olivier Muller ${ }^{a}$}

a Division of cardiology, Department of internal medicine, University Hospital of Lausanne (CHUV), Switzerland; ${ }^{b}$ Institute of pathology, University Hospital of Lausanne (CHUV), Switzerland; ' Department of radiology, University Hospital of Lausanne (CHUV), Switzerland; ${ }^{d}$ Division of cardiovascular surgery, Department of surgery, University Hospital of Lausanne (CHUV), Switzerland; e The Cecil Clinic, Hirslanden, Lausanne, Switzerland

\section{Summary}

Pulmonary artery sarcomas are uncommon intracardiac tumours with poor prognosis. We report the case of a 69 -year-old woman in good health presenting with rapidly progressive dyspnoea and an unfamiliar systolic murmur. Echocardiography revealed pulmonary valve stenosis due to an obstructing mobile mass. Imaging studies confirmed the presence of a contrast-enhancing lesion adherent to the valve, extending into the pulmonary trunk and right ventricular outflow tract, and suggestive of malignancy. Endovascular biopsy was attempted with no success. Surgical resection with autologous graft valve replacement and pulmonary artery reconstruction was performed. Postoperative histological examination confirmed the diagnosis of an undifferentiated pleomorphic sarcoma. Pulmonary artery sarcoma should be considered as a rare differential diagnosis in patients presenting with dyspnoea and a crescendo-decrescendo systolic murmur increasing with inspiration. Echocardiography is a useful first diagnostic approach but multi-imaging assessment is almost always necessary for definite diagnosis. Our case provides insights into the challenges met by cardiologists, radiologists and cardiac surgeons in the management of such cases.

Key words: pulmonary artery sarcoma; undifferentiated pleomorphic cardiac sarcoma; pulmonary valve stenosis; right ventricular outflow tract

\section{Case description}

A 69-year-old Caucasian woman presented to her general practitioner with new onset fatigue and rapidly progressive dyspnoea on exertion. Upon physical examination the patient was afebrile, blood pressure was $116 / 72 \mathrm{~mm} \mathrm{Hg}$, pulse rate $76 / \mathrm{min}$ and respiration rate $12 / \mathrm{min}$. Heart sounds were normal, but a new, 3/6, crescendo-decrescendo systolic mur- mur, which increased during deep inspiration and was maximal along the left sternal border, was noted. Laboratory studies of electrolytes, renal function, liver and cardiac enzymes, acute phase reactants and complete blood count were within normal limits, as were a 12-lead ECG and a chest radiograph.

Transthoracic echocardiography (TTE) revealed a moderate pulmonary valve stenosis caused by a globular mass protruding into the right ventricular outflow tract (RVOT) during diastole (fig. 1). The maximum pulmonary pressure gradient was measured at $42 \mathrm{~mm} \mathrm{Hg}$ (mean $30 \mathrm{~mm} \mathrm{Hg}$ ). Left as well as right ventricle size and function were normal.

The patient was promptly referred to our institution where cardiac computed tomography (CT) revealed a $25 \times 35 \times 24 \mathrm{~mm}$ mass attached to the RVOT and extending into the pulmonary trunk through the pulmonary valve (fig. 2). After intravenous injection of $60-80 \mathrm{ml}$ iodinated contrast material, late image acquisition showed inhomogeneous enhancement of the mass and mural enhancement of the pulmonary trunk suggestive of infiltration. This technique in the context of cardiac CT consists of acquiring images 60 seconds after $\mathrm{CT}$ angiography in order to optimise tissue enhancement. Fludeoxyglucose (18F) positron emission tomography-CT (18F-FDG PET-CT) revealed mild metabolic activity of the mass, with no signs of metastasis (fig. 3). Finally, cardiac magnetic resonance imaging (MRI) confirmed proximal extension of the valve mass, $4 \mathrm{~cm}$ towards the pulmonary trunk, without apparent invasion of the bifurcation (fig. 4). A hyperintense signal in T2-weighted images indicated oedema, whereas moderate signal-enhancement during resting perfusion suggested rich vascularisation. Intense signals on gadolinium en- 

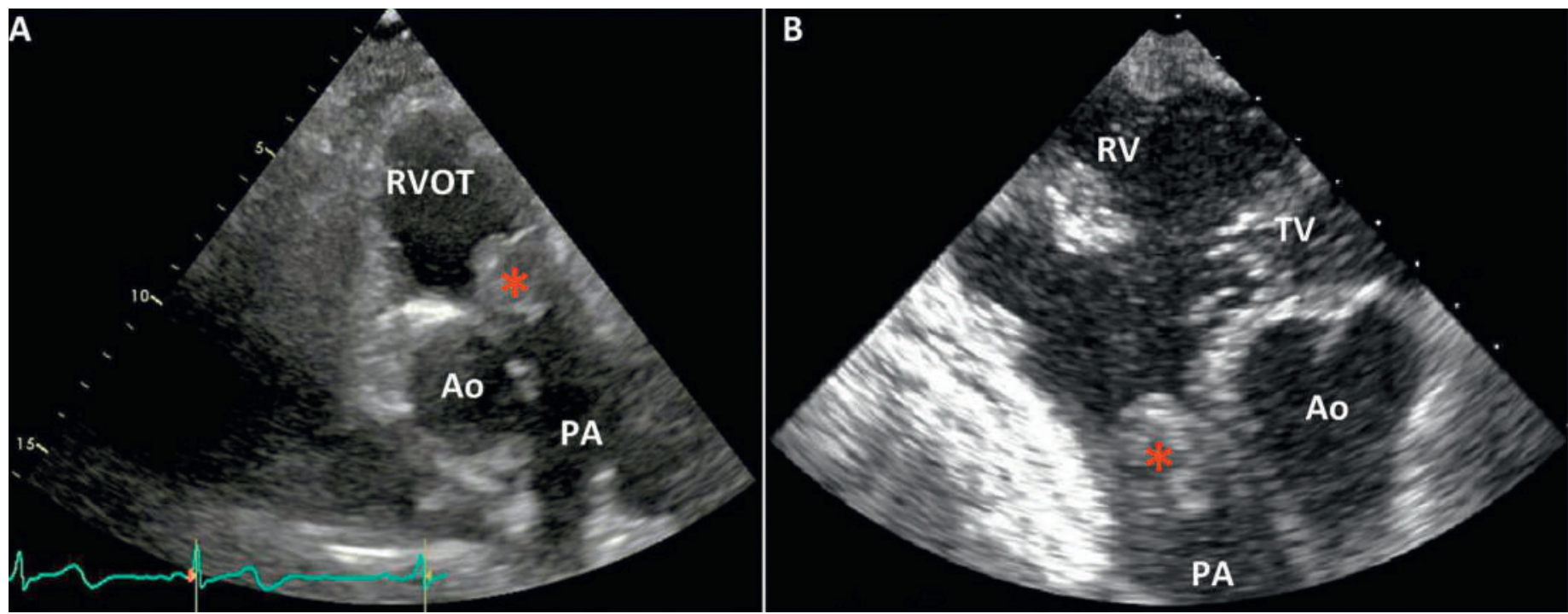

Figure 1: (A) TTE parasternal long axis view of the pulmonary artery. The tumour is recognised as a large round mass invading the pulmonary valve.

(B) Transoesophageal echocardiography (TOE) in transgastric inflow-outflow view of the right ventricle. The tricuspid valve and the right ventricular apex

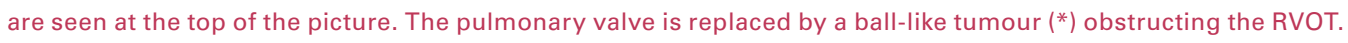
$\mathrm{Ao}=$ aorta; $\mathrm{PA}=$ pulmonary artery; $\mathrm{RV}=$ right ventricle; $\mathrm{RVOT}=$ right ventricle outflow tract; $\mathrm{TV}=$ tricuspid valve.

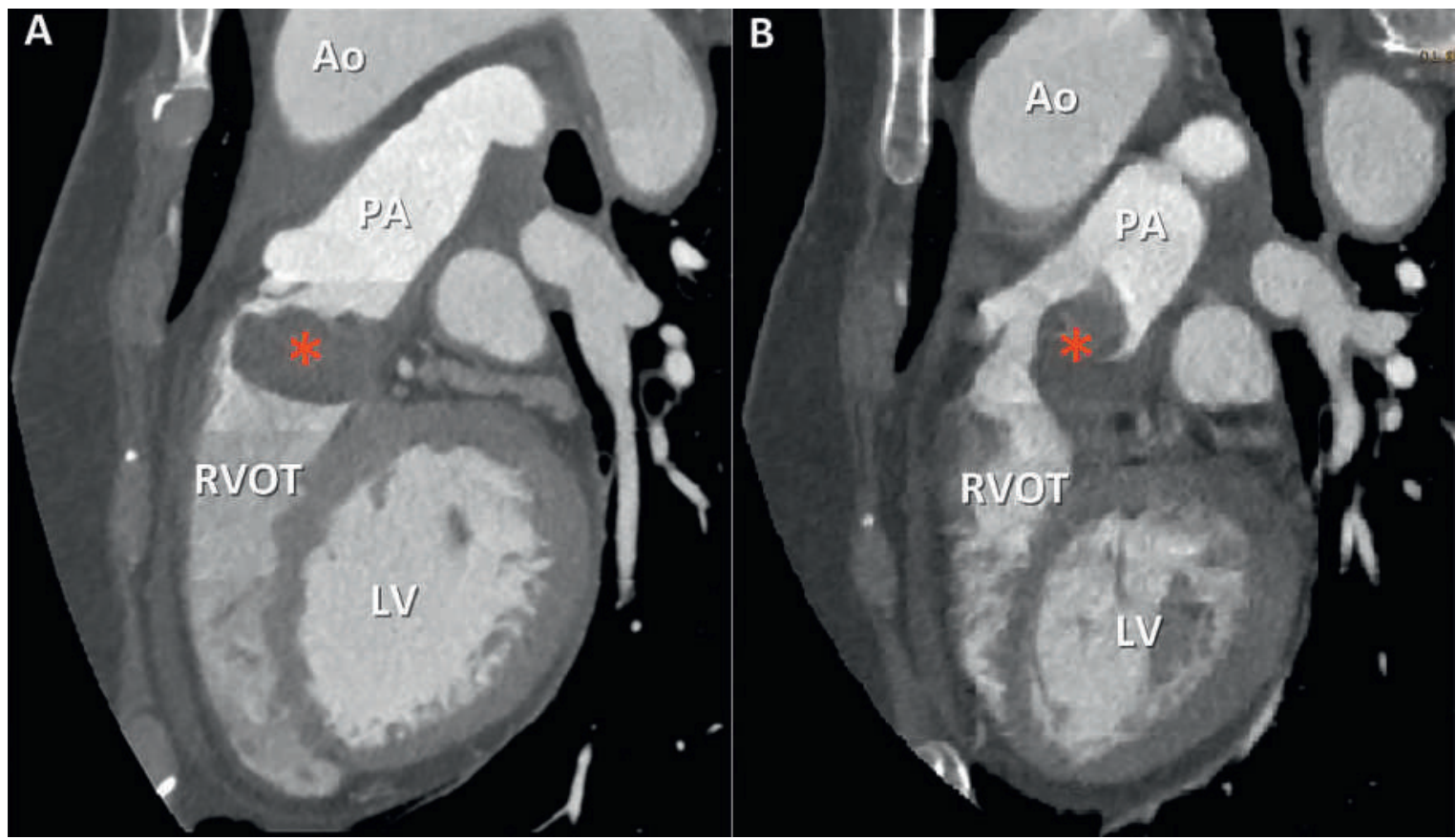

Figure 2: Cardiac computed tomography, $2 \mathrm{~d}$ multi-planar reformation showing the voluminous mass $\left({ }^{*}\right)$ attached to the pulmonary valve (A) and protruding in the pulmonary trunk during systole (B). $A o=$ aorta; $L V=$ left ventricle; $P A=$ pulmonary artery; $R V O T$ = right ventricle outflow tract.

hancement sequences suggested rich extracellular content (fibrosis or extracellular matrix), but no significant necrosis (fig. 5).

A provisory diagnosis of intracardiac malignancy was made on clinical (rapid installation of symptoms) and imaging (vascular infiltrative mass) grounds, and endovascular biopsy was attempted. The procedure was uneventful, but yielded limited material for histology. The case was discussed by a multidisciplinary panel involving cardiologists, radiologists and a cardiothoracic surgeon, and surgery was decided on. The time delay from first diagnosis 


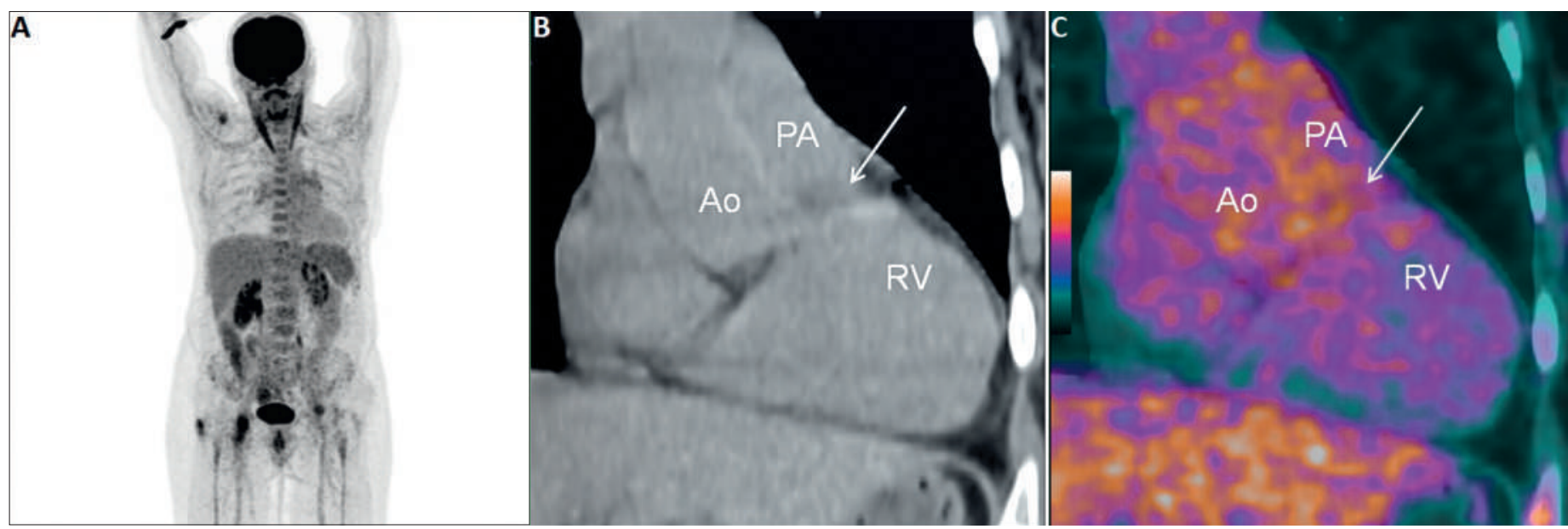

Figure 3: Fludeoxyglucose (18F) positron emission tomography-computed tomography (18F-FDG PET-CT) showing discrete metabolic activity of the mass (arrow), with no evidence of other lesion.

$\mathrm{Ao}=$ aorta; $\mathrm{PA}=$ pulmonary artery; $\mathrm{RV}=$ right ventricle.

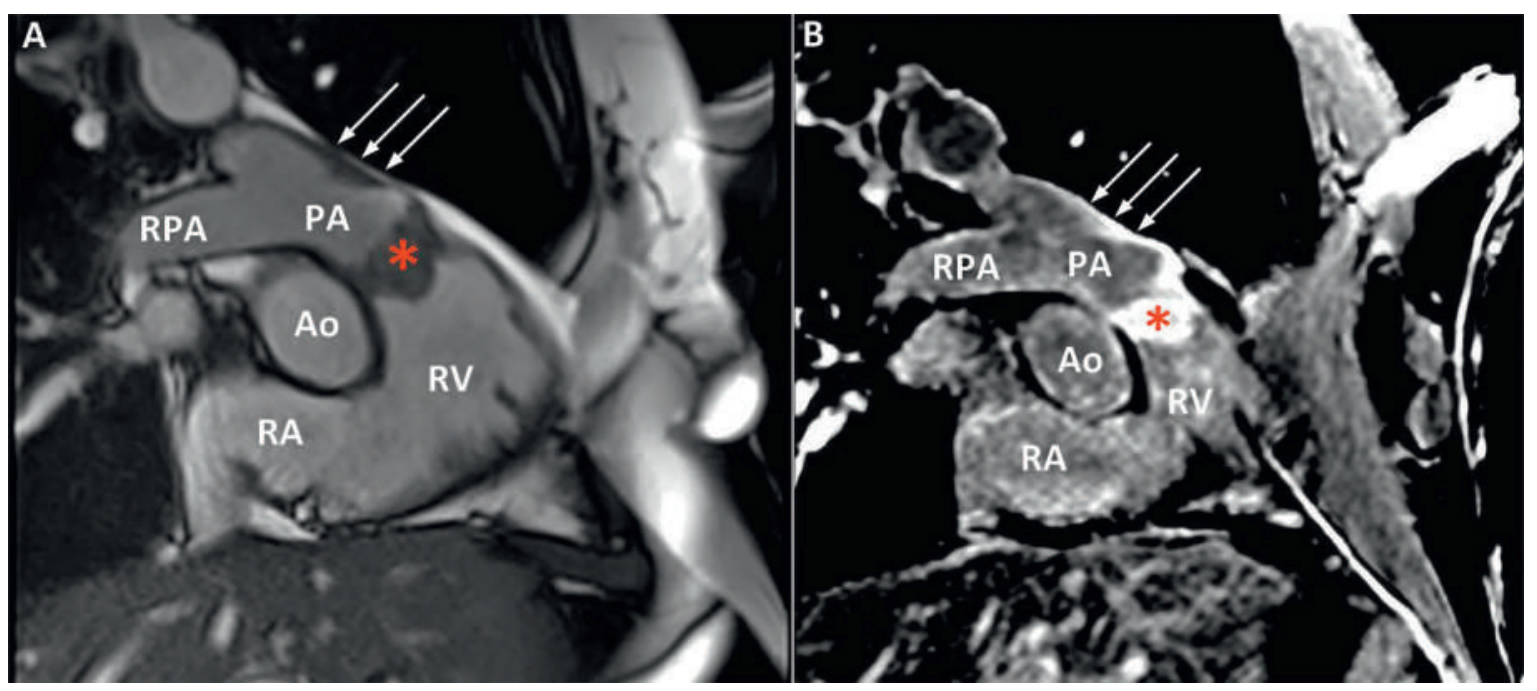

Figure 4: (A) Cardiac magnetic resonance imaging (MRI) steady-state free precession (SSFP) cine acquisition in inflow-outflow view of the right ventricle. The tumour is seen as a massive thickening of the pulmonary valve $(*)$. Note also the thickening of the anterior wall of the pulmonary trunk (arrows). (B) Corresponding acquisition with a phase-sensitive inversion-recovery sequence after contrast injection. There is homogenous late gadolinium enhancement of the tumour ${ }^{*}$ ) and of the anterior wall of the pulmonary trunk (arrows).

$\mathrm{Ao}=$ aorta; $\mathrm{PA}=$ pulmonary artery; $\mathrm{RPA}=$ right pulmonary artery; $\mathrm{RA}=$ right atrium; $\mathrm{RV}=$ right ventricle .

on TTE to the operation was 22 days. During the procedure, a bulky, white mass, densely adherent and infiltrating the pulmonary valve and all of the pulmonary infundibulum was identified and removed, along with the deformed pulmonary valve, part of the RVOT, as well as most of the pulmonary trunk as proximally as possible to the bifurcation. An autologous graft was used to replace the valve; the right ventricle was then anastomosed to the distal part of the pulmonary artery. Postoperative histology revealed a mesenchymal tumour consisting of highly atypical spindle cells without specific differentiation (fig. 6) [1]. Given the retrograde extension of the mass and the possibility of residual fragments very low inside the RVOT, the patient was referred for adjuvant chemotherapy and radiotherapy after six weeks of convalescence in a cardiac rehabilitation centre.

\section{Discussion}

Our case highlights the major impediments encountered during management of these rare and often 

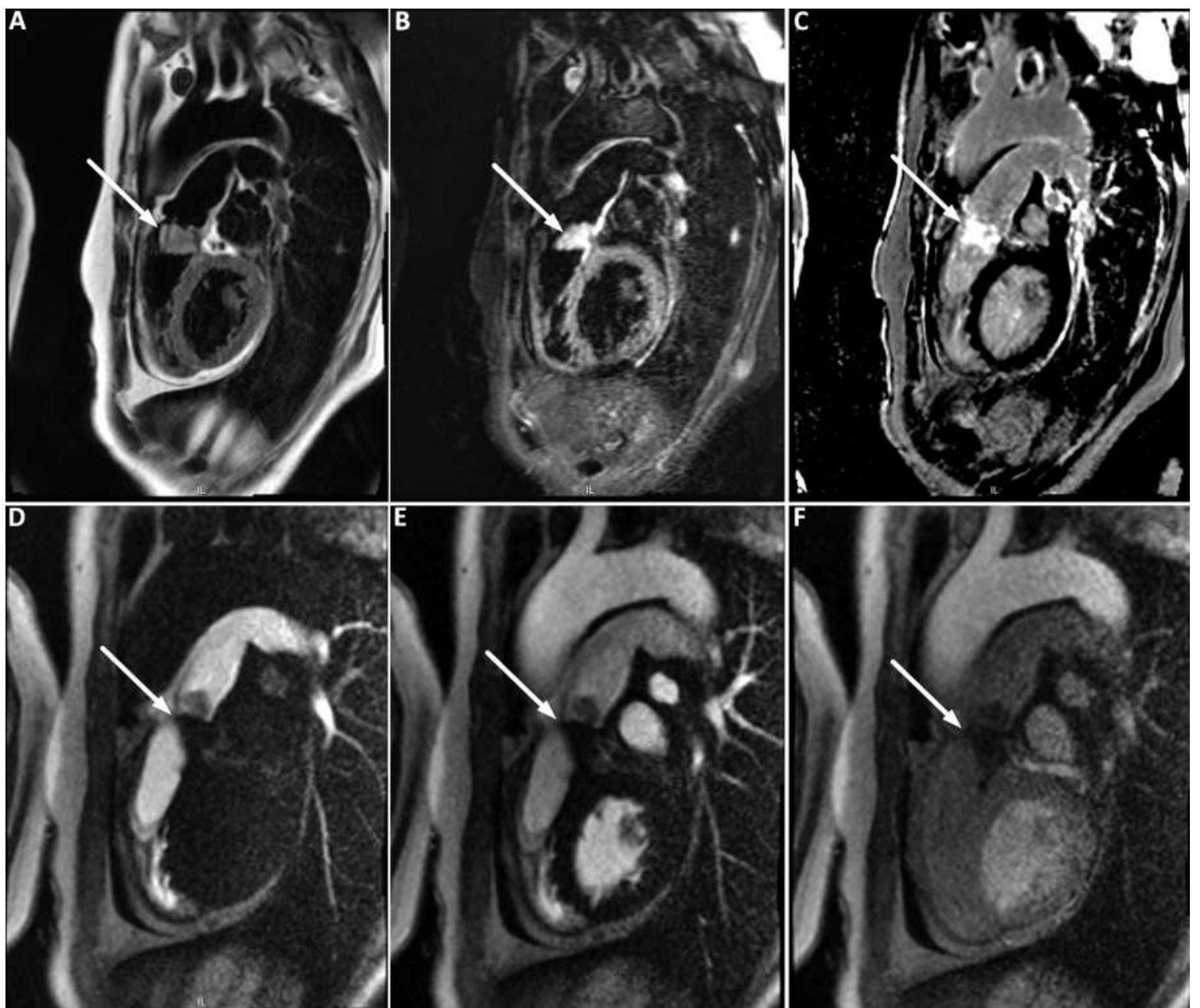

Figure 5: (A) T1-weighted image; the mass (arrow) has a signal iso-intense to the myocardium. (B) T2-weighted image; the mass has a signal hyper-intense to the myocardium indicating presence of tissue oedema. (C) Late gadolinium enhancement sequence; the mass shows bright enhancement indicating rich vascularisation. (D to F) Perfusion imaging; (D) contrast in the right cardiac chambers; (E) contrast in the left cardiac chambers; (F) contrast enhancing the myocardium: the mass shows moderate first-pass enhancement with peak signal intensity lower than that of the myocardium.

fatal intracardiac tumours; from timely and accurate diagnosis to surgical resection and adjuvant care, pulmonary artery sarcomas (PAS) represent a diagnostic and therapeutic challenge for cardiologists, radiologists, cardiac surgeons and oncologists.

Typical onset age is $45-55$ years with a female to male ratio of 2:1. The tumour typically arises within the pulmonary trunk and spreads into the pulmonary arteries. Embryologically, it originates from the bulbus cordis, a structure that later gives birth to both the RVOT and pulmonary trunk, which could, in our case, explain valve involvement and RVOT extension. As with the majority of intracardiac tumours, presentation is non-specific, with dyspnoea on exertion, chest or back pain, cough and haemoptysis being common symptoms. In many cases, the clinical pre- sentation mimics chronic thromboembolic pulmonary hypertension, and in advanced stages constitutional symptoms appear. Physical examination remains unremarkable until late. Our case is unique in the sense that it presented as a new unfamiliar murmur that alarmed the primary physician. Such a finding is extremely rare and highlights the paramount importance of good auscultation skills and a clinical acumen. A new harsh systolic murmur that increases with inspiration indicates pulmonary stenosis and warrants further investigation with echocardiography. TTE may show an increased gradient across a narrowed RVOT [2]. The presence of a pedunculated lesion arising from the RVOT, or pulmonary valve or trunk should always raise suspicion of PAS [3] and prompt a thoracic CT or a cardiac MRI. 

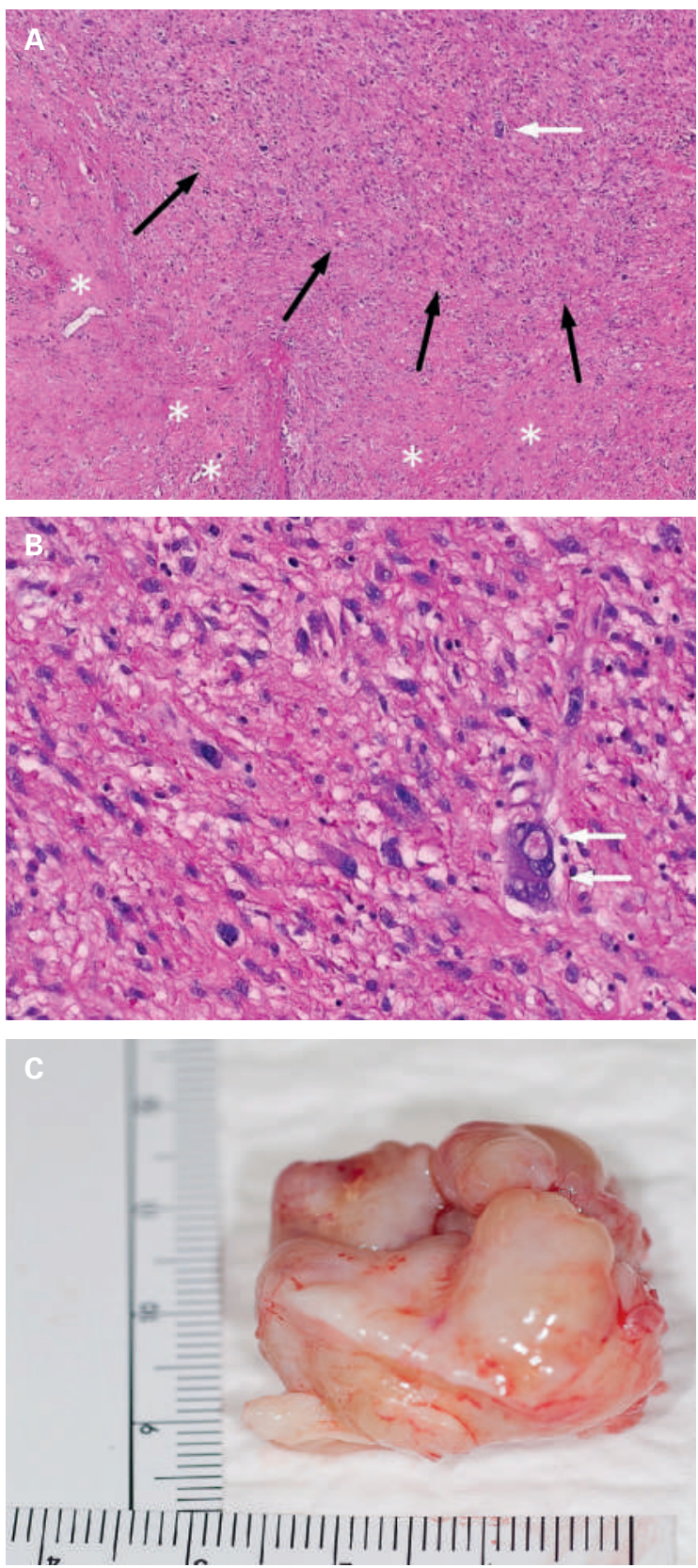

Correspondence: Panagiotis Antiochos, MD University Hospital of Lausanne (CHUV) 46 , rue du Bugnon CH-1011 Lausanne Switzerland panagiotis.antiochos [at]chuv.ch
Figure 6: (A) (Haematoxylin and eosin; $\times 40$ ) Postoperative histology revealed a poorly differentiated tumour composed of a proliferation of spindle cells with fibroblastic differentiation (black arrows). Some areas have a fibromyxoid pattern $\left({ }^{*}\right)$. (B) (haematoxylin and eosin; $\times 200$ ); Highly atypical giant spindle cell (white arrows). (C) Macroscopic aspect of the mass.
CT findings can be misleading in over $50 \%$ of cases, as PAS is confused with the far more common condition of central pulmonary embolism. Both entities present with a filling defect in the pulmonary arteries. Suggestive of PAS are an inhomogeneous, soft tissue density, with the filling defect occupying the entire lumen of the pulmonary trunk, along with delayed contrast enhancement on angiography [4]. Once suspicion is raised, PET-CT and MRI are helpful to differentiate fully the mass. Preoperative histological diagnosis is generally not possible although it should be attempted through CT-guided transthoracic aspiration, transvenous catheter biopsy or transbronchial biopsy. In most cases definitive diagnosis is made only during surgery or at autopsy. Surgery remains the cornerstone of management, offering definite diagnosis, clinical improvement and the best prognosis [5]. Even if curative resection is not possible, palliative resection can provide symptom relief by restoring haemodynamics. Reported interventions range from bilateral pneumonectomy, pulmonary endarterectomy (PEA) with or without pneumonectomy and with or without reconstruction of the pulmonary artery to debulking and palliative stenting. Regarding adjuvant therapy, combined chemoand radiotherapy should be adopted in nearly every case to extend palliation. Overall, PAS represents a fatal disease, with a mean survival time without surgical intervention of 1.5 months. Survival of $36.5 \pm 20.2$ months has been reported for patients benefiting from a "curative" resection while $11 \pm 3$ months for those undergoing "incomplete" resection [5].

In conclusion, it is important that physicians recognise the clinical features associated with PAS and include it as a differential diagnosis in the appropriate setting. Early referral for multidisciplinary management in a tertiary centre offers the only chance for prolonged survival and improved quality of life.

\section{Disclosures}

No financial support and no other potential conflict of interest relevant to this article was reported.

\section{References}

A full list of references is available in the online version of this article. 